\title{
Increased occurrence of native septic arthritis in adult cirrhotic patients: a population-based three-year follow-up study in Taiwan
}

\author{
Tsung-Hsing Hung ${ }^{1,2}$, Min-Hong Hsieh³, Chorng-Jang Lay ${ }^{2,4}$, Chih-Chun Tsai ${ }^{5}$, Chen-Chi Tsai ${ }^{2,4}$ \\ 'Division of Gastroenterology, Department of Medicine, Dalin Tzu Chi Hospital, Buddhist Tzu Chi Medical Foundation, Chiayi, \\ Taiwan \\ ${ }^{2} S c h o o l$ of Medicine, Tzu Chi University, Hualien, Taiwan \\ ${ }^{3}$ Department of Orthopedics, Dalin Tzu Chi Hospital, Buddhist Tzu Chi Medical Foundation, Chiayi, Taiwan \\ ${ }^{4}$ Division of Infectious diseases, Department of Medicine, Dalin Tzu Chi Hospital, Buddhist Tzu Chi Medical Foundation, Chiayi, \\ Taiwan \\ ${ }^{5}$ Department of Mathematics, Tamkang University, New Taipei City, Taiwan
}

Prz Gastroenterol 2014; 9 (6): 342-347

DOI: $10.5114 /$ pg.2014.47896

Key words: arthritis, cirrhosis, septic arthritis.

\begin{abstract}
Address for correspondence: Dr. Chen-Chi Tsai, Division of Infectious Disease, Department of Medicine, Dalin Tzu Chi Hospital, Buddhist Tzu Chi Medical Foundation, No. 2, Minsheng Rd., Dalin Township, Chiayi County 62247, Taiwan, phone: +886 5 2648000, fax: + 88652648999 , e-mail: antibody_1@msn.com
\end{abstract}

\begin{abstract}
Introduction: Due to impairment of immunity and metabolism, cirrhotic patients are prone to infection, osteoporosis, and osteonecrosis. However, it is unknown if cirrhotic patients are prone to native septic arthritis (NSA).

Aim: To assess the occurrence of NSA between cirrhotic and non-cirrhotic patients.

Material and methods: We used the Taiwan National Health Insurance Database to enrol 35,106 cirrhotic patients and 33,457 non-cirrhotic patients from January 1, 2004 to December 31, 2004. The medical record of each patient was individually followed for a 3-year period.

Results: There were $341(0.5 \%)$ patients having NSA in a follow-up period of 3 years: 214 cirrhotic and 127 non-cirrhotic patients. The incidence density of hospitalisation for NSA was 2.03 episodes/1000 person-years in cirrhotic patients, and 1.27 episodes/1000 person-years in non-cirrhotic patients. After adjustment for age, gender, and other comorbid disorders, Cox's regression analysis showed that cirrhotic patients had a higher occurrence of NSA than non-cirrhotic patients(hazard ratio $(\mathrm{HR})=1.51,95 \%$ confidence interval $(\mathrm{Cl})=1.19-1.90 ; p=0.001)$. The patients with complicated cirrhosis were more prone to have NSA than those with non-complicated cirrhosis ( $\mathrm{HR}=1.46,95 \% \mathrm{Cl}=1.09-1.96, p=0.011)$.

Conclusions: This analysis demonstrates that cirrhotic patients have a higher risk of NSA, particularly those with complicated cirrhosis.
\end{abstract}

\section{Introduction}

Septic arthritis, also called infectious arthritis, is inflammation of a joint caused by bacterial, viral, or fungal infection. The pathogens can enter into the joint capsule via direct penetration by traumatic injuries or surgical procedures, or via a haematogenous route $[1$ 2]. The yearly incidence of septic arthritis varies from 2 to 10 per 100,000 patients in the general population $[3,4]$. The incidence is doubled in the elderly, especially among those who have underlying medical conditions such as diabetes mellitus (DM), malignancy, chronic re- nal failure (CRF), rheumatoid arthritis (RA), alcoholism, stem cell transplant recipients, and acquired immune deficiency syndrome (AIDS) [2-12]. The affected joints for native septic arthritis (NSA) often have pre-existing damage, including traumatic injuries or degeneration $[1,9,10]$.

Cirrhosis is defined histologically as a diffuse hepatic process characterised by fibrosis and the conversion of normal liver architecture into structurally abnormal nodules. When patients have cirrhosis, they are prone to have variable infectious diseases, especially bacterae- 
mias [13]. This is attributed to intestinal bacterial overgrowth and translocation, impaired phagocytosis, poor opsonisation capacity, porto-systemic shunting, and low complement levels in cirrhotic patients [14-16]. In addition, cirrhotic patients are prone to have osteoporosis and osteonecrosis due to poor cholecalciferol absorption through the intestine, poor activation of vitamin D, chronic hypogonadism, and overexpression of interleukin-6 [17-19]. However, there has been no study to confirm that liver cirrhosis is a significant risk factor for NSA due to low incidence of NSA in the general population.

We used the Taiwan National Health Insurance Database to enrol a large population of cirrhotic patients and comparison patients. We followed the patients for a 3-year period to compare the occurrence of NSA in both groups. To the best of our knowledge, this is the first study to ascertain if cirrhosis is a risk factor for the occurrence of NSA.

\section{Material and methods \\ Database}

In 1995, the National Health Insurance (NHI) program started in Taiwan. Currently, the National Health Insurance Bureau (BNHI) covers more than $98 \%$ of the Taiwan population. All contracted medical institutions must provide medical records to the $\mathrm{BNHI}$ for medical payment of patient hospitalisation. According to the regulations governing the review of the medical services, the $\mathrm{BNHI}$ reviews reimbursement claims filed by contracted medical institutions and screens the type, volume, quality, and appropriateness of medical services provided under the NHI program. These medical records are established as a database, the National Health Insurance Research Database (NHIRD), which is maintained by the BNHI and the National Health Research Institute (NHRI). The dataset in the current study is from this database, which includes all diagnostic coding information of hospitalised patients in Taiwan. In order to protect the privacy of the patients, the researchers signed a guarantee to protect the patients' privacy. The present study was approved by the NHRI, and its agreement number is 99247.

\section{Study sample}

This is a retrospective cohort study. The discharged patients with a primary or accessory diagnosis of cirrhosis of the liver [International Classification of Diseases, 9th Revision, Clinical Modification (ICD-9-CM) code 571.2, or 571.5] from January 1, 2004 to December 31, 2004 were identified from the database. Cirrhotic patients who had had septic arthritis or received total hip replacement or total knee replacement before the time of enrolment for the following study were excluded. In
Taiwan National Health Insurance, cirrhotic patients can get a certification to reduce their payments for hospitalisation when they are diagnosed with refractory ascites, episodes of oesophageal/gastric variceal bleeding, or hepatic encephalopathy. When cirrhotic patients use certifications to reduce their payment for hospitalizations, we can identify them from the database. They were considered to have complicated cirrhosis [20, 21]. Patients less than 30 years of age were excluded because their causes of cirrhosis were often different from adult cirrhotic patients. Patients with missing or incomplete basic data were excluded. Patients with autoimmune disease-related cirrhosis, like primary biliary cirrhosis, autoimmune hepatitis, and primary sclerosing cholangitis, were excluded.

In total, 35,221 adult cirrhotic patients without baseline septic arthritis and prosthetic joints were enrolled in this study. The comparison group was composed of other patients in a database of 40,769 hospitalised non-cirrhotic patients. We excluded the patients with baseline septic arthritis or prosthetic joints. There were ten patients in the comparison group with incomplete basic data. The remaining 33,778 non-cirrhotic patients were selected as a comparison group. Individually, each patient was followed for a 3-year period starting at the time of the enrolment episode of hospitalisation, and continuing until the occurrence of NSA (ICD-9-CM codes 711) $[22,23]$. Patients who received total knee replacement or total hip replacement in their follow-up period, before diagnosis of septic arthritis, were excluded. There were 35,106 cirrhotic patients and 33,457 non-cirrhotic patients enrolled for the study.

The other risk factors for septic arthritis were counted if the condition was noted at the time of the hospitalisation before the end of the study, including alcoholism (ICD-9-CM codes 291, 303, 305.00-305.03, 571.0-571.3), DM (ICD-9-CM code 250), gout (ICD-9-CM codes 274), intravenous drug abuse (IVDA) (ICD-9-CM code 304), CRF (ICD-9-CM code 585), RA (ICD-9-CM code 714), solid organ transplantation (SOT) (ICD-9-CM codes V42.0, V42.1,V42.7), and systemic lupus erythematosus (SLE) (ICD-9-CM codes 710.0).

\section{Statistical analysis}

To determine the time to septic arthritis, survival time was defined as the time from the enrolment hospitalisation to the time of hospitalisation for septic arthritis, with patients censored at death, or the end of the study. We used the SPSS statistical package (SPSS System for Windows, version 13.0) to perform the analyses in this study. We used $\chi^{2}$ test to compare the variables between cirrhotic and non-cirrhotic patients. Stepwise regression (likelihood ratio method) was used 
to model all factors associated with time to hospitalised septic arthritis, controlling for age and all covariates. The potential confounding factors were entered into the regression model, including DM, IVDA, CRF, SLE, alcoholism, age groups, RA, Gout, SOT, and gender. Age groups were entered into the regression model as continuous co-variables using the following coding: $1=30-44$, $2=45-59,3=60-74$, and $4=\geq 75$ years. Finally, we presented hazard ratios (HR) along with the $95 \%$ confidence intervals $(\mathrm{Cl})$. A $p$ value of $<0.05$ was considered statistically significant.

\section{Results}

A total of 35,106 adult cirrhotic patients without baseline septic arthritis or prosthetic joints were enrolled in this study. The comparison group was composed of 33,457 non-cirrhotic patients without baseline septic arthritis or prosthetic joints. In total, there were $341(0.5 \%)$ patients with NSA in a follow-up period of 3 years: 214 cirrhotic and 127 non-cirrhotic patients. The incidence density of hospitalisation for septic arthritis was 2.03 episodes/1000 person-years in cirrhotic patients, and 1.27 episodes/1000 person-years in non-cirrhotic patients. Table I shows the distribution of demographic characteristics and selected confounding factors for NSA between cirrhotic and non-cirrhotic patients. The cirrhotic patients had significantly more patients with DM, alcoholism, and SOT. The non-cirrhotic patients had significantly more patients with gout, RA, and IVDA. Other factors including SLE and CRF were not different significantly between both groups.
After Cox proportional regression model adjusted by age, gender and other confounding factors, including SLE, DM, CRF, alcoholism, IVDA, alcoholism, RA and gout, the HR of cirrhosis for the occurrence of NSA in a 3-year follow-up period was 1.51 (95\% Cl: 1.19-1.90, $p=0.001$ ). The result for Cox's proportional regression model is shown in Table II. For NSA, the HR of DM was 1.51 (95\% Cl: 1.18-1.93; $p=0.001)$, SLE was $7.96(95 \% \mathrm{Cl}$ : $1.97-32.09 ; p=0.004)$, alcoholism was $1.67(95 \% \mathrm{Cl}$ : 1.27-2.25, $p<0.001)$, RA was 6.78 (95\% Cl: 2.52-18.24; $p<0.001)$, IVDA was 16.25 (95\% Cl: 4.03-65.49; $p<0.001$ ), CRF was 2.29 (95\% Cl: 1.49-3.54; $p>0.001$ ), and gout was 4.39 (95\% Cl: 3.05-6.31; $p<0.001)$.

Because the severity of cirrhosis could not be identified from our database, we selected refractory ascites, oesophageal/gastric variceal bleedings, and hepatic encephalopathy as the markers for severe cirrhosis. When the patients had had refractory ascites, episodes of oesophageal/gastric variceal bleeding, or episodes of hepatic encephalopathy, they were considered as having complicated cirrhosis. Of 35,106 cirrhotic patients, there were 7889 (22\%) patients with complicated cirrhosis and 27,217 (78\%) patients with non-complicated cirrhosis. The NSA occurred in $148(0.5 \%)$ of the non-complicated cirrhotic patients and 66 (0.8\%) of the complicated cirrhotic patients during a 3-year follow-up period. Compared with non-complicated cirrhosis, the crude HR of complicated liver cirrhosis for the occurrence of NSA in a period of 3 years was 1.54 (95\% Cl: 1.15-2.06; $p=0.003)$. After adjustment for gender, age group, and other confounding factors for NSA

Table I. Demographic characteristics of 35,106 cirrhotic patients and 33,457 comparison patients

\begin{tabular}{lccc} 
Parameter & $\begin{array}{c}\text { Cirrhotic patients } \\
(n=35106)\end{array}$ & $\begin{array}{c}\text { Non-cirrhotic patients } \\
(n=33457)\end{array}$ & Value of $p$ \\
\hline Age groups $n$ (\%) [years]: & $6067(17.3)$ & $5931(17.7)$ & 0.050 \\
\hline $30-44$ & $11266(32.1)$ & $10884(32.5)$ & $11291(33.7)$ \\
\hline $45-59$ & $12213(34.8)$ & $5351(16.0)$ & $>0.050$ \\
\hline $60-74$ & $5560(15.8)$ & $23959(71.6)$ & $<0.001$ \\
\hline$>75$ & $24949(71.1)$ & $5910(17.7)$ & 0.001 \\
\hline Male, $n(\%)$ & $6977(19.9)$ & $25(0.1)$ & $>0.050$ \\
\hline Diabetes mellitus, $n$ (\%) & $7(0.0)$ & $981(2.9)$ & 1.000 \\
\hline IVDA, $n(\%)$ & $1101(3.1)$ & $35(0.1)$ & $<0.001$ \\
\hline CRF, $n(\%)$ & $25(0.1)$ & $556(1.7)$ & $<0.001$ \\
\hline SLE, $n(\%)$ & $6775(19.3)$ & $88(0.3)$ & $<0.001$ \\
\hline Alcoholism, $n(\%)$ & $39(0.1)$ & $872(2.6)$ & $<0.001^{a}$
\end{tabular}

IVDA - intravenous drug abuser, CRF - chronic renal failure, SLE - systemic lupus erythematosus, RA - rheumatoid arthritis, SOT - solid organ transplantation. 
Table II. Adjusted hazard ratios of the risk factors for native septic arthritis over a period of 3 years

\begin{tabular}{lccc} 
Variable & Hazard ratio & 95\% confidence interval & Value of $p$ \\
\hline Cirrhosis & 1.51 & $1.19-1.90$ & 0.001 \\
\hline DM & 1.51 & $1.18-1.93$ & 0.001 \\
\hline Alcoholism & 1.67 & $1.27-2.25$ & $<0.001$ \\
\hline CRF & 2.29 & $1.49-3.54$ & $<0.001$ \\
\hline Gout & 4.39 & $3.05-6.31$ & $<0.001$ \\
\hline RA & 6.78 & $2.52-18.24$ & $<0.001$ \\
\hline SLE & 7.96 & $1.97-32.09$ & 0.004 \\
\hline IVDA & 16.25 & $4.03-65.49$ & $<0.001$
\end{tabular}

SLE - systemic lupus erythematosus, DM - diabetes mellitus, CRF - chronic renal failure, IVDA - intravenous drug abuser, RA - rheumatoid arthritis.

Table III. Crude and adjusted hazard ratios of complicated cirrhosis for native septic arthritis, compared to noncomplicated cirrhosis

\begin{tabular}{lccc} 
Development of native septic arthritis & Non-complicated cirrhosis, $\boldsymbol{n}(\%)$ & Complicated cirrhosis, $\boldsymbol{n}(\%)$ & Value of $\boldsymbol{p}$ \\
\hline Yes & $148(0.5)$ & $66(0.8)$ & \\
\hline No & $27069(99.5)$ & $7823(99.2)$ & \\
\hline Crude hazard ratio (95\% confidence interval) & 1 & $1.54(1.15-2.06)$ & 0.003 \\
\hline *Adjusted hazard ratio (95\% confidence interval) & 1 & $1.46(1.09-1.96)$ & 0.011
\end{tabular}

*Adjustments were made for patient's gender, age, diabetes mellitus, chronic renal failure, systemic lupus erythematosus, rheumatoid arthritis, gout, intravenous drug abuser, and alcoholism.

(i.e. DM, CRF, SLE, RA, gout, IVDA, and alcoholism), the HR of complicated cirrhosis was 1.46 (95\% Cl: 1.09-1.96; $p=0.011$ ). Table III summarises the crude and adjusted HRs of complicated cirrhosis for septic arthritis compared with non-complicated cirrhosis. Figure 1 shows the plot of time to occurrence of NSA among non-cirrhotic patients, complicated cirrhotic patients, and non-complicated cirrhotic patients.

\section{Discussion}

After adjustment for age, gender, and other comorbid disorders for NSA, the present study showed that the cirrhotic patients were prone to NSA, especially those with complicated cirrhosis. In the present study, the incidence densities of hospitalisation for NSA in cirrhotic and non-cirrhotic patients were much higher than previous data. This is because all of the patients enrolled in our study were inpatients, not members of the general population. There were more patients with underlying medical disorders predisposing to septic arthritis in the present study, so they had higher incidence of NSA.

Compatible with previous studies, DM, CRF, RA, SLE, IVDA, gout, and alcoholism are risk factors for occurrence of septic arthritis. The IVDAs had the highest HR for NSA. Numerous articles have drawn attention to the increased incidence of bone and joint infections in IVDAs $[24,25]$. The NSA is unusual in the non-IVDA population with infective endocarditis, despite relatively prolonged periods of continuous bacteraemia associated with infective endocarditis [25]. The predominance of Staphylococcus aureus as the pathogen responsible for

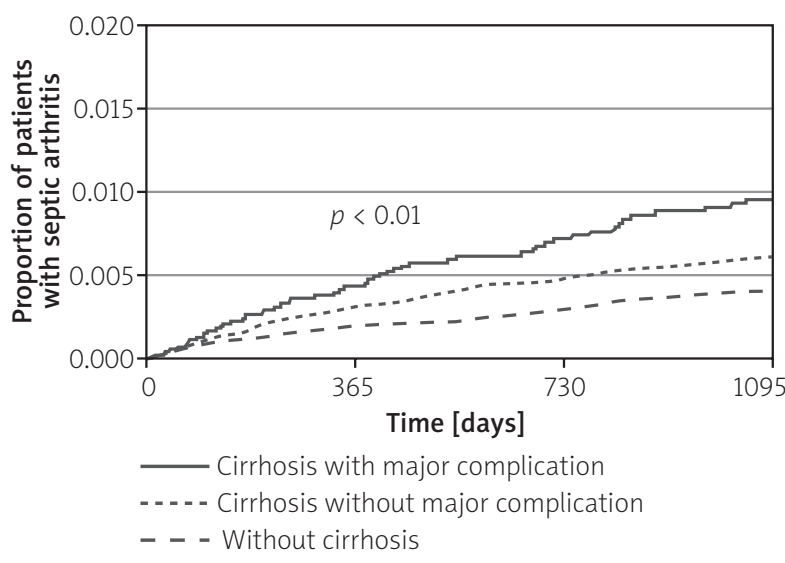

Figure 1. Time to hospitalisation for native septic arthritis in complicated cirrhotic patients $(n=7889)$, non-complicated cirrhotic patients $(n=27,217)$, and comparison patients $(n=33,457)$ over a period of 3 years. The proportions of complicated cirrhotic patients, non-complicated cirrhotic patients, and comparison patients with native septic arthritis over a period of 3 years were $0.84 \%, 0.54 \%$, and $0.38 \%$, respectively $(p<0.01)$ 
NSA in IVDAs may itself be responsible for the increased incidence of NSA in this population. Followed by IVDAs, the patients with the autoimmune diseases, including SLE and RA, had high HR for the occurrence of NSA. Compatible with previous studies, our study showed the risk of NSA in SLE and RA patients, irrespective of therapy, is increased $6-8$ fold $[26,27]$. In addition to the destructive joints caused by SLE or RA, the use of immunosuppressive medications played an important role for the occurrence of NSA. Literature reports about concomitant septic and gouty arthritis are rare [28]. However, in a study from Taiwan, a high proportion (43.5\%) of patients with septic ankles had concomitant gouty arthritis [29]. Our study proved the risk of NSA in gout patients was increased four fold. This may be attributed to joint destructions and steroid usage in the gout patients.

The HR of cirrhosis for the occurrence of NSA is similar to the HRs of DM, alcoholism, and CRF. Patients with these chronic disorders have impaired innate immunity. Sustained hyperglycaemia can retard neutrophil chemotaxis in DM patients [30]. Pre-activated peripheral blood CD14(+) leukocyte can impair innate immune response in CRF patients with haemodialysis [31]. Alcoholism can result in a LPS-mediated hypoinflammatory conditioning of the innate immune system [32, 33]. Similarly, cirrhotic patients had neutrophil phagocytic dysfunction [14-16]. The impairment of the innate immunity in these underlying conditions may be associated with increased occurrence of NSA.

There are some limitations worth mentioning in the present study. Firstly, the database used in this study did not included complete individual information such as dosage and duration of steroid use or the amount of alcohol consumed by patients. Secondly, the aetiology of cirrhosis could only be classified into alcohol related and non-alcohol related. However, the aetiology of cirrhosis in Taiwan was already well established in many previous studies, and it was shown to be related mostly to hepatitis B virus [34, 35]. In our study, about $80 \%$ of cirrhotic patients were non-alcoholic related, which was compatible with the data in Taiwan. Thirdly, it was not possible to identify the Child-Pugh score of cirrhosis by ICD-9 coding in the database. We could only classify the severity of cirrhosis into complicated and non-complicated cirrhosis according the diagnostic coding information. Fourthly, we cannot confirm the diagnostic tool used by the clinical physicians for septic arthritis because the database used in this study did not include the lab data. Definite diagnosis of septic arthritis is by synovial fluid culture. However, some physicians may use image study or leukocyte count of synovial fluid for diagnosis of septic arthritis. Different methods for diagnosis of NSA may contribute some bias. This is an unsolved problem in our study. Fifthly, the comparison group is not a healthy control group, so the hazard ratio of cirrhosis for NSA may be underestimated compared to a healthy population. Additionally, we cannot exclude the possibility of misclassification of cirrhosis or septic arthritis based on the database. We thought only a few misclassifications existed in our database and a large population can alleviate this effect.

Nonetheless, this study is the first complete, nationwide, population-based study to show a correlation between cirrhosis and native septic arthritis. This analysis demonstrates that cirrhotic patients have a higher risk of NSA, particularly those with complicated cirrhosis.

\section{Acknowledgments}

This study is based in part on data from the $\mathrm{Na}$ tional Health Insurance Research Database provided by the Bureau of National Health Insurance, Department of Health, Taiwan. The database is managed by the National Health Research Institute (Registered number: 101516). Interpretations and conclusions contained herein do not represent those of the Bureau of National Health Insurance, Department of Health, or the National Health Research Institute.

\section{References}

1. Jones A, Henderson MJ, Berman P, et al. Septic arthritis complicating apatite associated destructive arthropathy. Ann Rheum Dis 1990; 49: 1005-7.

2. Mathews CJ, Kingsley G, Field M, et al. Management of septic arthritis: a systematic review. Ann Rheum Dis 2007; 66: 440-5.

3. Shirtliff ME, Mader JT. Acute septic arthritis. Clin Microbiol Rev 2002; 15: 527-44

4. Kaandorp CJ, Dinant HJ, van de Laar MA, et al. Incidence and sources of native and prosthetic joint infection: a community based prospective survey. Ann Rheum Dis 1997; 56: 470-5.

5. Ho G Jr. Bacterial arthritis. Curr Opin Rheumatol 2001; 13: 310-4.

6. Margaretten ME, Kohlwes J, Moore D, et al. Does this adult patient have septic arthritis? JAMA 2007; 297: 1478-88.

7. Sharp JT, Lidsky MD, Duffy J, et al. Infectious arthritis. Arch Intern Med 1979; 139: 125-30.

8. al-Jarallah KF, Shehab DK, Buchanan WW. Rheumatic complications of alcohol abuse. Semin Arthritis Rheum 1992; 22: 162-71.

9. Vincent GM, Amirault JD. Septic arthritis in the elderly. Clin Orthop Relat Res 1990; 251: 241-5.

10. Goldenberg DL, Reed JI. Bacterial arthritis. N Engl J Med 1985; 312: 764-71.

11. Kelly PJ. Bacterial arthritis in the adult. Orthop Clin North Am 1975; 6: 973-81.

12. Clerc O, Prod'hom G, Greub G, et al. Adult native septic arthritis: a review of 10 years of experience and lessons for empirical antibiotic therapy. J Antimicrob Chemother 2011; 66: 1168-73. 
13. Garcia-Tsao G. Bacterial infections in cirrhosis. Can J Gastroenterol 2004; 18: 405-6.

14. Mowat C, Stanley AJ. Spontaneous bacterial peritonitis - diagnosis, treatment and prevention. Aliment Pharmacol Ther 2001; 15: 1851-9.

15. Tritto G, Bechlis Z, Stadlbauer V, et al. Evidence of neutrophi functional defect despite inflammation in stable cirrhosis. J Hepatol 2011; 55: 574-81.

16. Leber B, Mayrhauser $U$, Rybczynski M, et al. Innate immune dysfunction in acute and chronic liver disease. Wien Klin Wochenschr 2009; 121: 732-44.

17. Guichelaar MM, Kendall R, Malinchoc M, et al. Bone mineral density before and after OLT: long-term follow-up and predictive factors. Liver Transpl 2006; 12: 1390-402.

18. Mclnnes IB, Schett G. Cytokines in the pathogenesis of rheumatoid arthritis. Nat Rev Immunol 2007; 7: 429-42.

19. Hung $\mathrm{TH}, \mathrm{H}$ sieh $\mathrm{YH}$, Tsai CC, et al. Is liver cirrhosis a risk factor for osteonecrosis of the femoral head in adults? A population-based 3-year follow-up study. Intern Med 2011; 50: 2563-8.

20. Menon KV, Kamath PS. Managing the complications of cirrhosis. Mayo Clin Proc 2000; 75: 501-9.

21. Resnick RH. Portal hypertension and its complications - an overview. J Clin Gastroenterol 1980; 2: 259-67.

22. Luhmann JD, Luhmann SJ. Etiology of septic arthritis in children: an update for the 1990s. Pediatr Emerg Care 1999; 15: 40-2.

23. Abid N, Bhatti M, Azharuddin M, et al. Septic arthritis in a tertiary care hospital. J Pak Med Assoc 2006; 56: 95-8.

24. Scheidegger C, Zimmerli W. Infectious complications in drug addicts: seven-year review of 269 hospitalized narcotics abusers in Switzerland. Rev Infect Dis 1989; 11: 486-93.

25. Vyskocil JJ, Mcllroy MA, Brennan TA, et al. Pyogenic infection of the sacroiliac joint. Case reports and review of the literature. Medicine 1991; 70: 188-97.

26. Favero $M$, Schiavon F, Riato $L$, et al. Rheumatoid arthritis is the major risk factor for septic arthritis in rheumatological settings. Autoimmun Rev 2008; 8: 59-61.

27. Huang JL, Hung JJ, Wu KC, et al. Septic arthritis in patients with systemic lupus erythematosus: salmonella and nonsalmonella infections compared. Semin Arthritis Rheum 2006; 36: 61-7.

28. Yu KH, Luo SF, Liou LB, et al. Concomitant septic and gouty arthritis - an analysis of 30 cases. Rheumatology 2003; 42: 1062-6.

29. Lee $\mathrm{CH}$, Chen YJ, Ueng SW, et al. Septic arthritis of the ankle joint. Chang Gung Med J 2000; 23: 420-6.

30. Wilson RM. Neutrophil function in diabetes. Diabet Med 1986; 3: 509-25

31. Kim HW, Woo YS, Yang HN, et al. Primed monocytes: putative culprits of chronic low-grade inflammation and impaired innate immune responses in patients on hemodialysis. Clin Exp Nephrol 2011; 15: 258-63.

32. Suh YG, Jeong WI. Hepatic stellate cells and innate immunity in alcoholic liver disease. World J Gastroenterol 2011; 17 2543-51.

33. Frank J, Witte K, Schrödl W, et al. Chronic alcoholism causes deleterious conditioning of innate immunity. Alcohol Alcohol 2004; 39: 386-92
34. Hsu HC, Lin WS, Tsai MJ. Hepatitis-B surface antigen and hepatocellular carcinoma in Taiwan. With special reference to types and localization of HBsAg in the tumor cells. Cancer 1983; 52: 1825-32.

35. Tsai JF, Chang WY, Jeng JE, et al. Hepatitis C virus infection as a risk factor for non-alcoholic liver cirrhosis in Taiwan. J Med Virol 1993; 41: 296-300.

Received: 26.03.2014

Accepted: 5.09 .2014 